\title{
Lactococcus Species Central Line-Associated Bloodstream Infection in Pediatrics: A Case Series
}

\author{
Sarah E. Firmani ${ }^{1,2}$, Holly D. Maples ${ }^{1,2}$ and Archana Balamohan ${ }^{2,3 *}$ \\ ' Department of Pharmacy Practice, College of Pharmacy, University of Arkansas for Medical Sciences, Little Rock, AR, \\ United States, ${ }^{2}$ Arkansas Children's Hospital, Little Rock, AR, United States, ${ }^{3}$ Department of Pediatrics, Section of Pediatric \\ Infectious Diseases, University of Arkansas for Medical Sciences, Little Rock, AR, United States
}

OPEN ACCESS

Edited by:

Richard V. Goering,

Creighton University, United States

Reviewed by:

Ayşe Karaaslan,

Istanbul Kartal Dr. Lutfi Kirdar

Education and Research

Hospital, Turkey

Yonggon Cho

Jeonbuk National University,

South Korea

*Correspondence:

Archana Balamohan

abalamohan@uams.edu

Specialty section:

This article was submitted to Infectious Diseases-Surveillance,

Prevention and Treatment, a section of the journal

Frontiers in Medicine

Received: 26 October 2021

Accepted: 12 January 2022

Published: 03 February 2022

Citation:

Firmani SE, Maples HD and Balamohan A (2022) Lactococcus

Species Central Line-Associated Bloodstream Infection in Pediatrics: A Case Series. Front. Med. 9:802493.

doi: 10.3389/fmed.2022.802493
Lactococcus spp. is typically thought to be of low virulence and seldom considered pathogenic. Few cases of significant infections in children have been reported, all outside of the United States. There is also limited data on antimicrobial susceptibility testing for Lactococcus spp. We present three pediatric patients with central line bloodstream infections due to Lactococcus spp. between 2018 and 2020, along with a review of the pediatric literature.

Keywords: Lactococcus, central line-associated bloodstream infection (CLABSI), bacteremia, pediatric infection, antimicrobial susceptibility testing

Central venous catheters (CVC) are often essential in the pediatric population. A serious complication of central access is central line-associated blood stream infection (CLABSI), which is associated with morbidity, mortality, and financial burden. In the pediatric population, neonates, oncology, and intestinal failure patients are at greatest risk for CLABSIs (1). These infections are caused by a wide variety of pathogens that often vary based on patients' comorbidities. Historically, Lactococcus species have seldom been considered pathogenic in humans. However, cases of clinically significant Lactococcus infections have been reported (2-11). Few pediatric cases have been reported, all of which are from geographic regions outside the United States. In this case series, we present three pediatric Lactococcus bacteremia cases from 2018 to 2020, all thought to be CLABSI. Informed consent was not obtained for this series of case reports, as patient identifiers are not included and their anonymity and confidentiality are protected.

\section{CASE 1}

A 203 day-old, 25-week gestational age infant, during his birth admission in the neonatal intensive care unit, developed a fever to 38.8 degrees Celsius. His medical conditions included bronchopulmonary dysplasia and an ileostomy due to intestinal perforation, requiring parenteral nutrition through his CVC. The remainder of his vitals were normal, and his physical examination was unrevealing. Central and peripheral blood cultures were obtained. He was started on empiric vancomycin and piperacillin-tazobactam therapy. After $8 \mathrm{~h}$ of incubation, his central blood culture grew gram-positive cocci in pairs and chains as well as gram-positive bacilli. The patient's CVC was removed. Three days after initial growth, two organisms were identified: Lactococcus lactis spp. lactis and Lactobacillus spp. His initial and three subsequent peripheral blood cultures remained negative.

When the species were identified, the patient's antibiotic therapy was deescalated to vancomycin monotherapy and the patient completed 10 days from first negative blood culture. Antimicrobial susceptibility testing was performed at a reference laboratory. These resulted 2 days after completion of his 
treatment, revealing susceptibility to ceftriaxone [MIC (minimum inhibitory concentration) $0.5 \mathrm{mcg} / \mathrm{mL}$, penicillin (MIC $0.5 \mathrm{mcg} / \mathrm{mL}$ ) and vancomycin (MIC $<0.5 \mathrm{mcg} / \mathrm{mL}$ ).

\section{CASE 2}

A 21-month-old term male with congenital nephrotic syndrome, stage 2 chronic kidney disease, and hypogammaglobulinemia with a CVC since 15 months of age, presented to the nephrology clinic for his biweekly albumin and furosemide infusions. During the albumin infusion, he became febrile to 38.5 degrees Celsius. He was subsequently admitted to rule out a CLABSI. Apart from chronic cough and congestion, the child was well-appearing with no alternate focus of infection.

Central and peripheral blood cultures were obtained prior to initiating empiric antibiotic therapy with cefepime. A respiratory viral panel was also obtained, which detected rhinovirus/enterovirus and adenovirus. After $14 \mathrm{~h}$ of incubation, the central blood culture grew gram-positive cocci in pairs and chains, at which point vancomycin was added to the antibiotic regimen. The organism was subsequently identified as Lactococcus lactis and was sent to a reference laboratory for antimicrobial susceptibility testing.

The peripheral blood culture obtained prior to antibiotics, as well as two repeat central blood cultures post antibiotics revealed no growth. The CVC was retained. Antimicrobial susceptibility results returned on day 8 of therapy, revealing susceptibility to ceftriaxone (MIC $0.5 \mathrm{mcg} / \mathrm{mL}$ ), penicillin (MIC $0.5 \mathrm{mcg} / \mathrm{mL}$ ), and vancomycin (MIC $0.25 \mathrm{mcg} / \mathrm{mL}$ ). Antibiotic therapy was deescalated to ceftriaxone monotherapy. He completed a total of 10 days of antibiotic therapy from first negative blood culture.

\section{CASE 3}

A 2-year-old male with a history of Hirschsprung's Disease and short bowel syndrome presented to an outside facility with fever and headache for 1 day. He was dependent upon Gastric-tube feedings and total parenteral nutrition, for which he had a CVC. Four weeks prior to presentation, his CVC had been repaired due to a leak. At the outside facility, his CVC was found to be occluded, and he was transferred to our hospital. On admission, physical exam was remarkable for a febrile (39.4 degrees Celsius), ill-appearing child with tachypnea, rhinorrhea, cough, and rales bilaterally. Crusting was noted adjacent to his ostomy site. An abdominal ultrasound was unrevealing.

A peripheral blood culture was drawn by the outside facility, after which he received a dose of cefepime. After alteplase was administered at our facility, a central line blood culture was obtained and patient was started on cefepime, vancomycin, metronidazole, and fluconazole. On day 2, the peripheral blood culture from the outside facility grew gram-positive cocci in chains. On day 3, the organism was identified as Enterococcus spp. On day 4, it was discovered that the isolate was misidentified, and was confirmed to be Lactococcus garvieae. Antimicrobial therapy was subsequently de-escalated to vancomycin monotherapy. An echocardiogram was performed and was negative for intracardiac vegetations. The isolate was sent to a reference laboratory for antimicrobial susceptibility testing and resulted on day 8 of admission as susceptible to ceftriaxone (MIC $0.5 \mathrm{mcg} / \mathrm{mL}$ ), penicillin (MIC $1 \mathrm{mcg} / \mathrm{mL}$ ), and vancomycin (MIC $0.5 \mathrm{mcg} / \mathrm{mL}$ ), at which point therapy was further narrowed to ceftriaxone. Repeat blood cultures (two central and one peripheral) remained negative. The CVC was retained. He completed a total of 14 days of antibiotic therapy from first negative blood culture.

\section{DISCUSSION}

Lactococcus is a gram-positive coccus, facultative anaerobe (12) consisting of eight species (8). Previously, Lactococcus was classified as Streptococcus, and often is misidentified as Enterococcus (12), as demonstrated by the third case. L. garvieae is commonly known to cause disease in farmed fish (12), and $L$. lactis is often used as an additive in food products (3). Lactococcus spp. has also been documented as being part of human intestinal normal flora $(3,4)$. Despite Lactococcus spp. historically being considered non-pathogenic, there have been reports of infections in adult and pediatric patients due to Lactococcus species in recent years.

Lactococcus species, like other organisms typically regarded as contaminants or non-pathogenic, have the potential to cause clinical infection in children at risk, including those with CVCs. In this case series, we report three pediatric cases with Lactococcus bacteremia, all CLABSI. The terminology used to define intravascular catheter-related infections, CLABSI and catheter-related bloodstream infection (CRBSI) are used interchangeably, although their meanings differ. CLABSI is a surveillance definition used to identify a primary bloodstream infection that is unrelated to an infection at another site in a patient with a CVC. CRBSI is a clinical definition that requires fulfillment of criteria in order to attribute the catheter as the source of the bloodstream infection (13). Confirming CRBSI in the pediatric population is challenging, as collecting peripheral blood cultures with sufficient volume of blood can be difficult (1) and colony-forming unit (CFU) and quantitative cultures of catheter-tip, central and peripheral blood cultures are seldom performed. Although Lactococcus species were not isolated from both central and peripheral cultures in our patients, we believe all infants had CLABSI due to rapid growth, fever at the time of infusion and/or absence of alternative explanation for fever. Per the National Healthcare Safety Network (NHSN), all our Lactococcus species cases met criteria for CLABSI-I, none of which fulfilled mucosal barrier injury-I criteria (14). Cases 1 and 2 were present on admission. Case 3 was a healthcare associated infection and was reported to NHSN.

We reviewed the literature of pediatric infections secondary to Lactococcus species and found 11 additional cases (Table 1). There are a greater number of reported pediatric infections due to $L$. lactis compared to L. garvieae $(1,8)$. The most common source of infection is blood (45\%), with $60 \%$ (three of five) occurring in patients with central lines. Other sources include liver abscess (9\%), brain abscess (9\%), cerebrospinal fluid (9\%), urine $(9 \%)$, and endocarditis (18\%). Median patient age 
TABLE 1 | Cases of Lactococcus spp. infections in pediatric patients (<18 years of age) in English, peer-reviewed literature since 2000 in PubMed database.

\begin{tabular}{|c|c|c|c|c|c|c|c|c|}
\hline References & Year, location & Age & Possible risk factors & Species & Source & $\begin{array}{l}\text { CVC } \\
\text { removed }\end{array}$ & $\begin{array}{l}\text { Antimicrobial therapy; } \\
\text { treatment duration }(d)\end{array}$ & $\begin{array}{l}\text { Susceptibility } \\
\text { interpretation and MICs* } \\
\text { (mcg/mL unless specified) }\end{array}$ \\
\hline Nakarai et al. (6) & 2000, Japan & 14 years & None & Lactococcus lactis & Liver abscess & $\mathrm{N} / \mathrm{A}$ & $\begin{array}{l}\text { CTM, AMK, CLN, PAN, PIP; } \\
35\end{array}$ & $\begin{array}{l}\text { 3+: PIP, CTM, ERY, CLN, } \\
\text { MIN, PAN } \\
\text { 1+: AMK }\end{array}$ \\
\hline Glikman et al. (2) & 2010, Israel & 9 months & $\begin{array}{l}\text { SBS } \\
\text { TPN } \\
\text { CVC }\end{array}$ & L. lactis & $\begin{array}{l}\text { Blood (central and } \\
\text { peripheral sources) }\end{array}$ & No & CFO, VAN; 10 & $\begin{array}{l}\text { S: PEN (0.25), CEF (0.125), } \\
\text { VAN (0.5) }\end{array}$ \\
\hline Topcu et al. (8) & 2011, Turkey & 19 months & $\begin{array}{l}\text { Ingestion of raw milk } 13 \\
\text { months prior }\end{array}$ & L. lactis & Brain abscess & $\mathrm{N} / \mathrm{A}$ & CEF, VAN, MER; 40 & Not reported \\
\hline Uchida et al. (9) & 2011, Japan & 16 days & Prematurity (36 weeks GA) & L. lactis & CSF & $\mathrm{N} / \mathrm{A}$ & CFO; 12 & $\begin{array}{l}\text { S: AMP, PEN, CFO, CFP, } \\
\text { ERY, CLA, CLN, VAN, LEV, } \\
\text { CHL, MER }\end{array}$ \\
\hline Newby et al. (7) & 2014, Canada & 14 days & $\begin{array}{l}\text { Prematurity ( } 31 \text { weeks GA) } \\
\text { Contaminated maternal breast } \\
\text { milk (L. lactis) }\end{array}$ & L. lactis & Urine & $\mathrm{N} / \mathrm{A}$ & CFO; 7 & $\begin{array}{l}\text { S: CFO, VAN, MER, AMO- } \\
\text { CLA } \\
\text { I: PEN, CEP } \\
\text { R: CFX, AMO }\end{array}$ \\
\hline Karaaslan et al. (3) & 2014, Turkey & 1 year & $\begin{array}{l}\text { Down syndrome } \\
\text { Hirschsprung's Disease TPN } \\
\text { CVC }\end{array}$ & L. lactis & $\begin{array}{l}\text { Blood (central and } \\
\text { peripheral) }\end{array}$ & No & VAN; 10 & S: VAN \\
\hline Mitra N, et al. (5) & 2015, India & 3 years & $\begin{array}{l}\text { Gingivitis, Stomatitis, R cervical } \\
\text { lymphadenitis, Fish and } \\
\text { unpasteurized milk intake }\end{array}$ & I Lactococcus garvieae & Blood & $\mathrm{N} / \mathrm{A}$ & AMO; not reported & S: AMO \\
\hline $\begin{array}{l}\text { Taniguchi et al. } \\
\text { (10) }\end{array}$ & 2015, Japan & 4 months & None & L. lactis & Endocarditis & $\mathrm{N} / \mathrm{A}$ & None (patient deceased) & Not reported \\
\hline $\begin{array}{l}\text { Karaaslan et al. } \\
\text { (11) }\end{array}$ & 2016, Turkey & 5 months & lleostomy & L. lactis & Blood (peripheral) & $\mathrm{N} / \mathrm{A}$ & VAN; 24 & $\begin{array}{l}\text { S: VAN (0.5) } \\
\text { I: PEN }(0.5)\end{array}$ \\
\hline $\begin{array}{l}\text { Karaaslan et al. } \\
\text { (11) }\end{array}$ & 2016, Turkey & 6 months & $\begin{array}{l}\text { TPN } \\
\text { CVC }\end{array}$ & L. lactis & Blood (central) & Yes & VAN; 10 & S: VAN (0.5), PEN \\
\hline Mansour et al. (4) & 2016, Israel & 11 years & None & L. lactis & Endocarditis & $\mathrm{N} / \mathrm{A}$ & CFA, CEF; 29 & Not reported \\
\hline $\begin{array}{l}\text { Presented Case } \\
\# 1\end{array}$ & $\begin{array}{l}\text { 2018, } \\
\text { United States }\end{array}$ & 6 months & $\begin{array}{l}\text { SBS } \\
\text { TPN } \\
\text { CVC }\end{array}$ & L. lactis & Blood (central) & Yes & VAN; 10 & $\begin{array}{l}\text { S: PEN (0.5), CEF (0.5), VAN } \\
(0.5):\end{array}$ \\
\hline $\begin{array}{l}\text { Presented Case } \\
\# 2\end{array}$ & $\begin{array}{l}\text { 2020, } \\
\text { United States }\end{array}$ & 21 months & $\begin{array}{l}\text { Congenital nephrotic syndrome } \\
\text { Stage II CKD } \\
\text { Hypogammaglobulinemia } \\
\text { CVC }\end{array}$ & L. lactis & Blood (central) & No & CEF; 10 & $\begin{array}{l}\text { S: PEN (0.5), CEF (0.5), VAN } \\
(0.25)\end{array}$ \\
\hline $\begin{array}{l}\text { Presented Case } \\
\# 3\end{array}$ & $\begin{array}{l}\text { 2020, } \\
\text { United States }\end{array}$ & 2 years & $\begin{array}{l}\text { SBS } \\
\text { Hirschsprung's Disease TPN } \\
\text { CVC }\end{array}$ & L. garvieae & Blood (peripheral) & No & CEF; 14 & $\begin{array}{l}\text { S: PEN (1), CEF (0.5), VAN } \\
(0.5)\end{array}$ \\
\hline
\end{tabular}

"If reported.

AMK, amikacin; AMO, amoxicillin; AMO/CLA, amoxicillin-clavulanate; AMP, ampicillin; CEF, ceftriaxone; CEP, cephalexin; CFA, cefazolin; CFO, cefotaxime; CFP, cefepime; CFX, cefixime; CHL, chloramphenicol; CKD, chronic kidney disease; CLA, clarithromycin; CLN, clindamycin; CSF, cerebrospinal fluid; CTM, cefotiam; CVC, central venous catheter; ERY, erythromycin; GA, gestational age; I, intermediate; LEV, levofloxacin; MER, meropenem; MIN, minocycline; PAN, panipenem; PEN, penicillin; PIP, piperacillin; R, resistant; S, susceptible; SBS, short bowel syndrome; TPN, total parenteral nutrition; VAN, vancomycin. 
was 12 months (range, 14 days-14 years). Six of 11 patients (54\%) had an underlying risk factor. Duration of therapy ranged from 7 to 40 days depending on the source of infection. Most definitive treatment regimens consisted of a third-generation cephalosporin (45\%). Of BSIs, $80 \%$ (four of five) of infants received vancomycin as part of their definitive therapy.

To the best of our knowledge, this series highlights the first reported pediatric cases of Lactococcus species infections in the United States. Our pediatric cases add to the scarce pool of existing data. All of our patients were in the infant-toddler age group, which is similar to international pediatric cases of Lactococcus BSIs (Table 1). The definitive source of Lactococcus bacteremia in these patients is not known. None of our patients were receiving probiotics. Two of our 3 cases did not take oral feedings, making dietary risk factors unlikely. Similar to the reported cases in Table 1 without known dietary risk factors, 2 of the 3 BSIs occurred in children with short bowel syndrome and all had the presence of a CVC, suggesting that bacterial translocation may be an inciting factor for these cases of CLABSI. Lactococcus species have been isolated from the gastrointestinal tract and may be a commensal organism that is part of the normal flora (4). One of our patients (Case 2) had a known immunodeficiency of hypogammaglobulinemia. However, none of the reported cases in Table 1 noted an immunodeficiency, suggesting that immune status is not a contributing factor to Lactococcus infections. Two of our 3 patients were treated for a total duration of 10 days, and two of our three patients were managed with CVC retention without recurrence of infection. This is consistent with the management of pediatric Lactococcus bloodstream infections in the literature (Table 1).

Isolates were identified using mass spectrometry. Antimicrobial susceptibility was performed by gradient diffusion and interpreted according to The Clinical Laboratory Standards Institute (CLSI) guidelines. CLSI has published susceptibility breakpoints for Lactococcus spp., including penicillin (MIC $\leq$ $1)$, ceftriaxone (MIC $\leq 1)$, and vancomycin (MIC $\leq 2)(15)$. Susceptibility testing for this organism is typically performed

\section{REFERENCES}

1. Janum S, Zingg W, Classen V, Afshari A. Bench-to-bedside review: challenges of diagnosis, care and prevention of central catheter-related bloodstream infections in children. Crit Care. (2013) 17:238. doi: 10.1186/cc12730

2. Glikman D, Sprecher H, Chernokozinsky A, Weintraub Z. Lactococcus lactis catheter-related bacteremia in an infant. Infection. (2010) 38:1456. doi: 10.1007/s15010-009-9378-2

3. Karaaslan A, Soysal A, Sarmiş A, et al. Lactococcus lactis catheter-related bloodstream infection in an infant: case report. Jpn J Infect Dis. (2015) 68:341-2. doi: 10.7883/yoken.JJID.2014.137

4. Mansour B, Habib A, Asli N, Geffen Y, Miron D, Elias N. A case of infective endocarditis and pulmonary septic emboli caused by Lactococcus lactis. Case Rep Pediatr. (2016) 2016:1024054. doi: 10.1155/2016/1024054

5. Mitra N, Kumar P. Lactococcus garvieae: an emerging pathogen. Indian Pediatr. (2015) 52:814.

6. Nakarai T, Morita K, Nojiri Y, Nei J, Kawamori Y. Liver abscess due to Lactococcus lactis cremoris. Pediatr Int. (2000) 42:699-701. doi: 10.1046/j.1442-200x.2000.01294.x

7. Newby B, Ramesh KK. Urinary tract infection in a preterm neonate caused by Lactococcus lactis. Can J Hosp Pharm. (2014) 67:453-4. doi: 10.4212/cjhp.v67i6.1409 at reference laboratories, which can have a longer turnaround time (7-12 days for our reported cases). Seven of 11 previously published cases reported quantitative antimicrobial sensitivity testing (AST) to beta-lactam antibiotics, of which $6(85 \%)$ were susceptible (Table 1). All three of our reported cases were susceptible to penicillin, ceftriaxone, and vancomycin, adding further AST data to the limited literature. The continued accrual of antimicrobial susceptibility data may bring forth the possibility of empirically treating Lactococcus spp. bacteremia with a 3rd generation cephalosporin.

In conclusion, we report three pediatric Lactococcus spp. CLABSI, two of which were successfully treated with CVC retention. These cases add to the limited literature regarding this organism-previously thought to be of low pathogenicity in humans-and its AST, suggesting that Lactococcus spp. should be considered pathogenic in the appropriate circumstances.

\section{DATA AVAILABILITY STATEMENT}

The original contributions presented in the study are included in the article/supplementary material, further inquiries can be directed to the corresponding author/s.

\section{AUTHOR CONTRIBUTIONS}

SF, AB, and HM conceptualized and designed the study, drafted the initial manuscript, and reviewed and revised the manuscript. All authors approved the final manuscript as submitted and agree to be accountable for all aspects of the work.

\section{FUNDING}

Funding for open access publishing was provided by Departmental Cabe Funding of the University of Arkansas for Medical Sciences.

8. Topçu Y, Akinci G, Bayram E, Hiz S, Türkmen M. Brain abscess caused by Lactococcus lactis cremoris in a child. Eur J Pediatr. (2011) 170:16035. doi: 10.1007/s00431-011-1586-3

9. Uchida Y, Morita H, Adachi S, Asano T, Taga T, Kondo N. Bacterial meningitis and septicemia of neonate due to Lactococcus lactis. Pediatr Int. (2011) 53:119-20. doi: 10.1111/j.1442-200X.2010.03240.x

10. Taniguchi K, Nakayama $M$, Nakahira $K$, et al. Sudden infant death due to lactococcal infective endocarditis. Leg Med. (2016) 19:10711. doi: 10.1016/j.legalmed.2015.07.013

11. Karaaslan A, Soysal A, Kepenekli Kadayifci E, et al. Lactococcus lactis spp lactis infection in infants with chronic diarrhea: two cases report and literature review in children. J Infect Dev Ctries. (2016) 10:304-7. doi: 10.3855/jidc. 7049

12. Gibello A, Galán-Sánchez F, Blanco MM, Rodríguez-Iglesias M, Domínguez L, Fernández-Garayzábal JF. The zoonotic potential of Lactococcus garvieae: an overview on microbiology, epidemiology, virulence factors, and relationship with its presence in foods. Res Vet Sci. (2016) 109:59-70. doi: 10.1016/j.rvsc.2016.09.010

13. O'Grady NP, Alexander M, Burns LA, Dellinger EP, Garland J, Heard SO, et al. Guidelines for the prevention of intravascular catheterrelated infections. Clin Infect Dis. (2011) 52:e162-93. doi: 10.1093/cid/ $\operatorname{cir} 257$ 
14. U.S. Centers for Disease Control and Prevention. The National Healthcare Safety Network (NHSN) Manual: 2022 NHSN Patient Safety Component Manual. Atlanta, GA: Division of Healthcare Quality Promotion, National Center for Emerging and Zoonotic Infectious Diseases (2022). Retrieved from: https://www.cdc.gov/nhsn/pdfs/pscmanual/pcsmanual_current.pdf

15. CLSI. Methods for Antimicrobial Dilution and Disk Susceptibility Testing of Infrequently Isolated or Fastidious Bacteria. 3rd ed. CLSI Guideline M45. Wayne, PA: Clinical and Laboratory Standards Institute (2016).

Conflict of Interest: The authors declare that the research was conducted in the absence of any commercial or financial relationships that could be construed as a potential conflict of interest.
Publisher's Note: All claims expressed in this article are solely those of the authors and do not necessarily represent those of their affiliated organizations, or those of the publisher, the editors and the reviewers. Any product that may be evaluated in this article, or claim that may be made by its manufacturer, is not guaranteed or endorsed by the publisher.

Copyright $\odot 2022$ Firmani, Maples and Balamohan. This is an open-access article distributed under the terms of the Creative Commons Attribution License (CC BY). The use, distribution or reproduction in other forums is permitted, provided the original author(s) and the copyright owner(s) are credited and that the original publication in this journal is cited, in accordance with accepted academic practice. No use, distribution or reproduction is permitted which does not comply with these terms. 